\title{
Wavelet and receiver operating characteristic analysis of heart rate variability
}

\author{
G. McCaffery, ${ }^{1}$ T. M. Griffith,,${ }^{2,3}$ K. Naka, ${ }^{3}$ M. P. Frennaux, ${ }^{3}$ and C. C. Matthai ${ }^{1}$ \\ ${ }^{1}$ Department of Physics and Astronomy, University of Wales, Cardiff, United Kingdom \\ ${ }^{2}$ Department of Diagnostic Radiology, University Hospital of Wales, Cardiff, United Kingdom \\ ${ }^{3}$ Welsh Institute of Heart Research, University Hospital of Wales, Cardiff, United Kingdom
}

(Received 17 September 2001; published 15 January 2002)

\begin{abstract}
Multiresolution wavelet analysis has been used to study the heart rate variability in two classes of patients with different pathological conditions. The scale dependent measure of Thurner et al. was found to be statistically significant in discriminating patients suffering from hypercardiomyopathy from a control set of normal subjects. We have performed Receiver Operating Characteristc (ROC) analysis and found the ROC area to be a useful measure by which to label the significance of the discrimination, as well as to describe the severity of heart dysfunction.
\end{abstract}

DOI: 10.1103/PhysRevE.65.022901

PACS number(s): 87.19.Hh

In recent years there has been growing interest in applying techniques from nonlinear dynamical systems theory to biological, physiological, and ecological systems. Many of the techniques involve the analysis of a time series. Cardiovascular signals, and in particular the interbeat $(R-R)$ time intervals, provide an easily accessible time series and as such have been the focus of much attention. Heart rate variability (HRV) exists even in the absence of physical or mental stress and may be considered a normal feature of healthy patients. There are many irregularities in this variability which are thought to be due to nonlinearities in the control network which in turn are principally determined by the autonomic nervous system and circulating humoral agents. In healthy (normal) subjects, it has been suggested that nonlinear dynamics appears to give a reasonable description of the heart rate [1-3]. Under pathological conditions, for example in congestive heart failure, there is a reduction in the complexity of the HRV. There is some evidence to suggest that the cardiac vagal activity is responsible for this complexity and hence any loss in complexity may be attributed to dysfunction of the cardiac system. It has also been suggested that detailed nonlinear analyses of the heart rate variability could lead to a diagnosis of pathological conditions. In such circumstances, it would be useful to have some measure by which such a diagnosis could be made.

Recent studies have focussed on identifying a reliable measure by which normal and pathological subjects may be differentiated. In this vein, Ivanov et al. [4] suggested that the probability distribution function of the $R-R$ intervals, after wavelet analysis and suitable scaling, can provide just such a measure. More recently, Thurner et al. [5] identified a HRV measure by which the condition of a cardiovascular system could be assessed. This measure is obtained by first performing a multiresolution wavelet analysis of the HRV time series, which decomposes the signal into its components at the different scales. By using wavelets, the nonstationarity effects inherent in the signal are removed [4]. Wavelets also have the property of acting like a "mathematical microscope" since by varying the scale of the wavelet, one can focus on different features of the dynamics. Using this measure, Thurner et al. [5] demonstrated that the standard deviation of the wavelet coefficients $\sigma_{w}(s)$ was a good scale $(s)$ dependent measure which could be used to discriminate between heart failure and normal subjects. Following on this work, Nunes Amaral et al. [6] proposed a scale-independent measure obtained from the scaling of the partition function of the wavelet coefficients. They also claimed this to be an effective discriminant of pathological conditions. It must be noted that all of these studies concentrated on patients suffering from quite serious heart problems which would be expected to be manifest in the HRV.

The success of these studies led Maronne et al. [7] to investigate the usefulness of these measures in studying other physiological time series and different pathological states. They found that by applying the standard deviation measure on the time series of systolic blood pressure waves maxima, it was indeed possible to differentiate between normal subjects and those with a condition called vasovagal syncope. Following this line of thought, we have also attempted to determine the range of the usefulness and applicability of these methods. We have considered two different diseased states, both of which may be attributed to dysfunction of the autonomic nervous system. As such these pathologies might be expected to be reflected in the HRV distributions. The results of these studies forms the content of this paper.

Patients with the condition termed hypertrophic cardiomyopathy (HCM) exhibit abnormal autonomic function resulting in an increased risk of sudden death with exercise. Although there may be distinct abnormalities in the electrocardiogram (ECG) of patients with HCM, as a result of intrinsic myocardial abnormalities (e.g., in the form of the QRS complexes and repolarization phases), we are primarily interested in detecting differences in the HRV which is determined principally by the autonomic nervous system and circulating humoral agents. ECG measurements were taken from two classes of patients: (a) a control group of 14 healthy subjects, selected from a large group and carefully screened for previous heart problems, with normal heart rate and blood pressure; (b) a group of 13 subjects with genetically determined HCM. The two groups were approximately age and gender matched. The data were acquired using the BIOPAC AcqKnowledge III system and was sampled at 10 -ms intervals with 16-bit resolution. Because of the in- 
creased risk to HCM patients when exercising, any differences in the HRV might be expected to be exaggerated in this state. They and the control group were examined while at rest and during exercise. Under resting conditions, each subject lay supine on a bed, while exercise was performed on a semierect cycle, with progressive work load increments (3min. stages) to a symptom limited maximum.

We have also studied the pathological condition of vasovagal syncope (VS) which was the subject of investigation by Marrone et al. [7]. This condition is thought to be due to a dysfunction of the autonomic nervous system which can result in a blackout when the patient experiences a sudden stimulus like the shift in blood volume around the body. This is especially manifest in a sudden vertical tilt of a patient initially lying horizontally. Instead of examining the time series of blood pressure maxima as was done by Marrone et al., we have again studied the time series of the $R-R$ intervals. For all the subjects, both normal and those with a tendency to VS, the ECG was measured with them lying down in a horizontal position and with them in a tilted position $60^{\circ}$ from the horizontal.

In both of these pathological states, the condition of the patients are expected to deteriorate under the appropriate external stimuli (exercise and tilt). These subjects might therefore also be expected to display a changed HRV pattern from that in the rest state.

Once the time series of the $R-R$ intervals, $a(i)$, was extracted from the ECG signals, a wavelet analysis was carried out. In this approach, the time series is expanded in a wavelet basis set. The wavelet coefficients are given by

$$
w_{s, n}=2^{-s / 2} \sum_{i=0}^{T-1} a(i) \psi\left(2^{-s} i-n\right),
$$

where $s$ is the wavelet scale, $n$ the interbeat number (which is an integer), and $T$ the number of samples. $\psi$ represents the mother wavelet. In order to discount the effects of nonstationarity and other variations, different mother wavelets (the Haar and the Daubechies 4, 12, and 20 coefficient filters) were considered. The sequence of wavelet coefficient standard deviation $\sigma_{w}(s)$ is determined through the equation [5]

$$
\sigma_{w}(s)=\left(\frac{1}{T-1} \sum_{n}\left[w_{s, n}-\bar{w}_{s, n}\right]^{2}\right)^{1 / 2},
$$

where $\bar{w}_{s, n}$ is the mean wavelet coefficient.

The first set of calculations were carried out for the HCM subjects and for the corresponding control set of normal subjects, under both rest and exercise conditions. In Fig. 1, the $\sigma_{w}$, thus obtained, are plotted as a function of scale for the Daubecies-20 coefficient wavelet. The results for the other wavelets, although differing in the details, were found to be very similar and so provided little new information for the purposes of this paper. Under rest conditions, it is clear that both sets of subjects display very similar spreads of $\sigma_{w}$ as well as covering the same range of values. Because of this overlap between the two sets of results, there is no discrimination between HCM and normal subjects when both groups are at rest. There is, however, a significant level of discrimi-
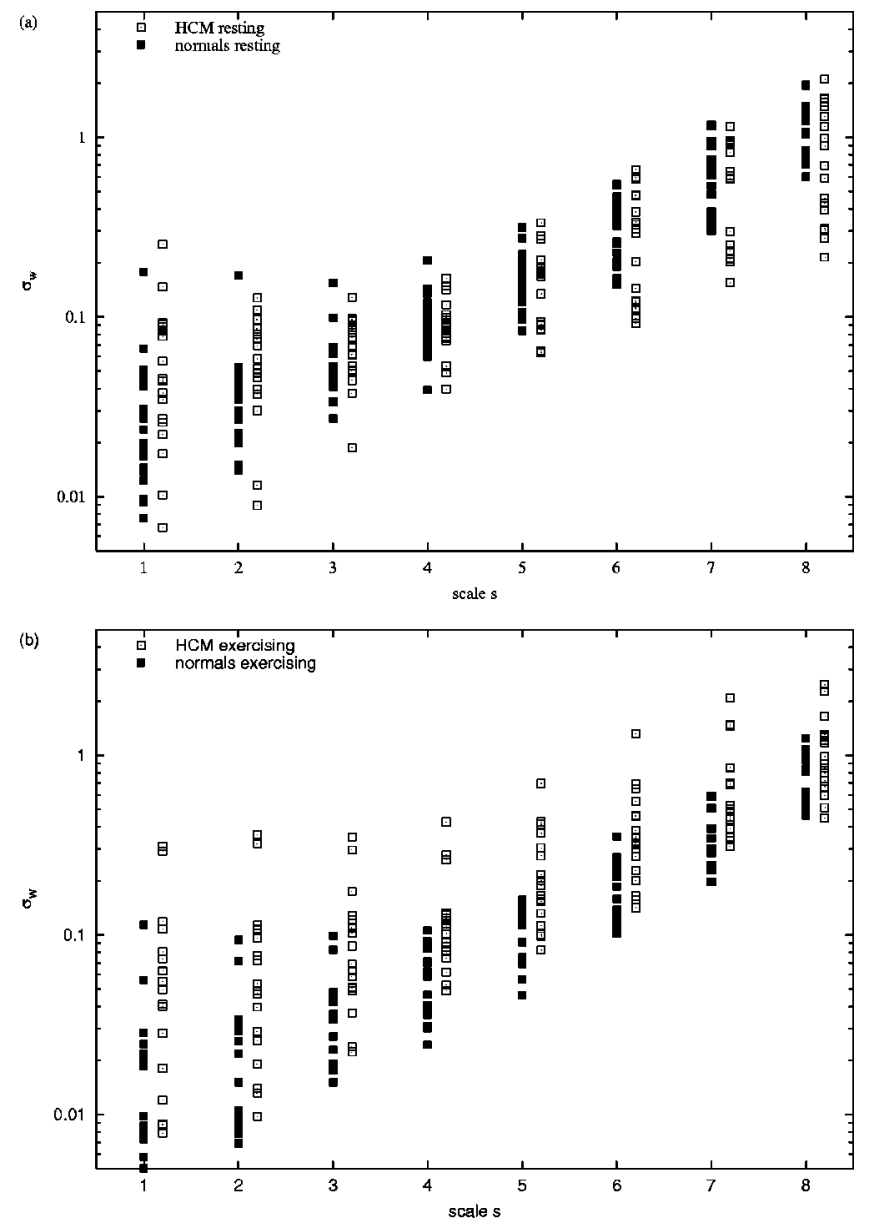

FIG. 1. The variation of the standard deviation of the wavelet coefficients $\sigma_{w}$ with wavelet scale $s$ for normal and HCM subjects under (a) rest and (b) exercise conditions.

nation between the two groups while exercising. The normal group has a similar spread to that found when they are at rest, although the average value of $\sigma_{w}$ is slightly lower. By contrast, the $\sigma_{w}$ of the HCM subjects display a greater spread in their values. Unlike that found for the heart failure subjects by Thurner et al., there is no complete separation of the two data sets. However, we too find that the discrimination appears to be best for scales $s=4$ and $s=5$. In order to test the statistical significance of these results, we carried out a Wilcoxon-Mann-Whitney (WMW) rank test. This tests the hypothesis that data from two sample sets belong to the same distribution. Thus, in our study, we pose the the question of whether the HRV distribution of the HCM patients are statistically the same as those of the normal subjects. The WMW is the appropriate choice of a nonparametric test when confronted with non-Gaussian data sets of relatively small size. From the results of the rank test on the pair of data sets determined during exercise conditions, we find that there is a probability of less than $10^{-3}$ that both sets of samples (normal and HCM) are drawn from the same continuous distribution function. By contrast, the WMW rank test on the two groups while resting gives a critical value for the sum of ranks indicating that the probability that the two data sets belong to the same distribution function is greater 


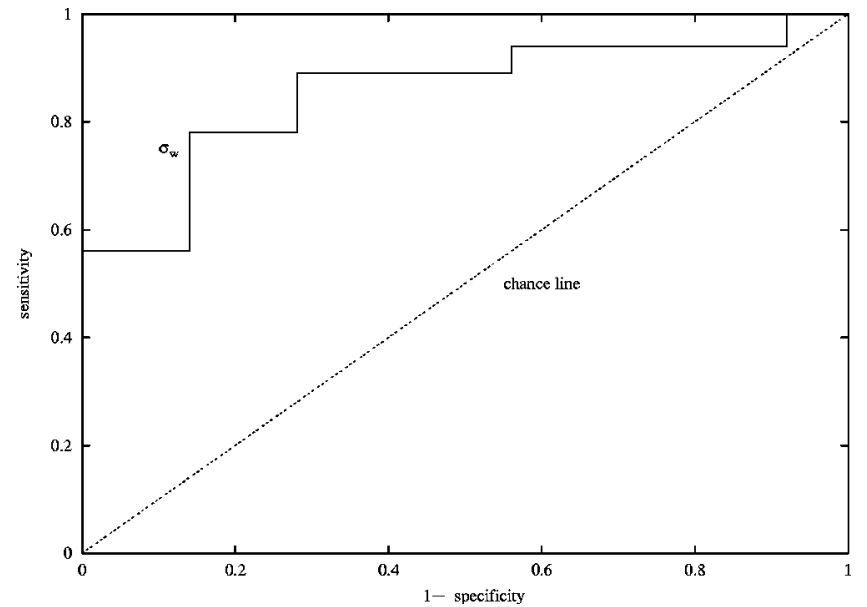

FIG. 2. Histogram showing the ROC curve of the $\sigma_{w}$ measure for the $\mathrm{HCM} /$ normal comparison under exercise conditions.

than 0.1 . This is not statistically significant and so allows us to conclude that the HRV distributions of HCM and normal subjects are the same while resting, but significantly different when they are exercising.

Receiver operating characteristic (ROC) analysis is a good method for assessing the suitability of a measure in binary hypothesis testing $[8,9]$. It provides another way of testing the hypothesis that two distributions are different. The ROC curve is obtained by plotting the proportion of pathological subjects being correctly identified (called the sensitivity) against a specified proportion of normal subjects being incorrectly diagonosed ( 1 - specificity). ROC analysis is particularly useful for quantifying the overlap between false negatives and false positives. Pure chance would result in a line along the diagonal with a ROC area (the area under the ROC curve) of about 0.5 . The maximum ROC area of 1.0 would correspond to disjoint distributions. Thus the ROC area provides a single number measure of the difference between two distributions.

Thurner et al. [10] (TFLT) used this appoach in their analysis of the HRV measure $\sigma_{w}$ of their earlier study [5]. We have also chosen to apply this method as it provides a means of comparing between different pathological states. This is of particular importance due to the overlap in the range of $\sigma_{w}$ values for the HCM and normal subjects. Following the approach of TFLT, we also determined that ROC curves for those wavelet scales for which the overlap was least $(s=4$ and $s=5)$. The result, for $s=5$ under exercise conditions, is shown in Fig. 2. We have used the same definition of the axes as TFLT, viz. the vertical axis is the sensitivity, indicating the propotion of HCM patients being correctly identified, and the horizontal axis is (1 - specificity) which is the proportion of control subjects falsely identified. From the figure, it is clear that the ROC curve is well away from the chance line [8]. The area under the ROC curve shown in Fig. 2 is approximately 0.86 confirming the results of the WMW test that the HRV distributions for the HRV and normal subjects while exercising are significantly different. By contrast, the ROC curve for the two sets under rest conditions is a zigzag line closely following the chance line. The
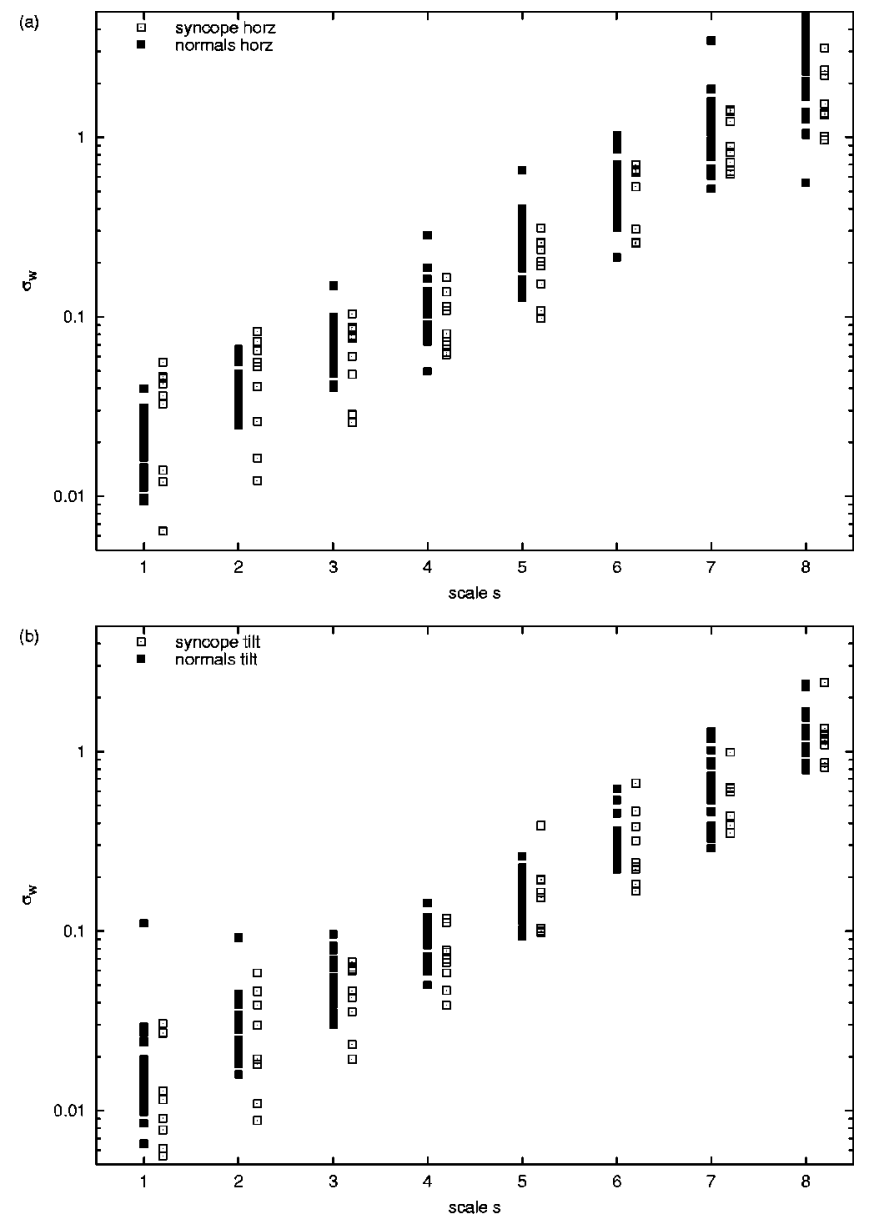

FIG. 3. The variation of the standard deviation of the wavelet coefficients $\sigma_{w}$ with wavelet scale $s$ for normal and VS subjects in (a) horizontal rest and (b) tilt configurations.

area under the ROC curve in this instance is only slightly greater than 0.5. This supports the rank test results but gives a better numerical measure to the discrimination.

Similar analyses were conducted for the group of subjects with VS and for their normal counterparts. The discrimination between the two sets of results in both horizontal (rest) and inclined states are less good than for the HCM subjects. However, in both tilted and nontilted states, there appears to be some measure of discrimination from the WMW rank test. For example, at wavelet scale $s=5$, the probability of concurrence is 0.01 . Interestingly, the spread of $\sigma_{w}$ for the syncope subjects is significantly less than that for the normal set at this wavelet scale (Fig. 3). It is surprising to note that there is no significant difference between the results obtained when the subjects were horizontal or tilted. A ROC curve analysis of the data gives slightly different results. For scale $s=5$, we find that the area under the ROC curve for the two sets while resting is 0.6 but reduces to only 0.5 when tilted. These results lead us to believe that VS is not manifest in the HRV.

In summary, we have performed a wavelet analysis of the interbeat interval time series from two classes of patients. We conclude that (i) $\sigma_{w}$ is a good measure to use in comparing the HRV of pathological conditions with those of normal 
subjects; (ii) ROC curve analysis is a very useful method by which to assess the suitability of HRV measures. In particular, the fact that the ROC area of the HCM pathological state changes between rest and exercise conditions supports the view that there is a correlation between ROC area and severity of cardiac dysfunction. Thus HRV is significantly differ- ent in HCM patients only while exercising. However, the results of the VS patients indicate that this measure may not be used as a discriminant for just any pathological condition.

G.McC. acknowledges financial support from the EPSRC.
[1] L. Glass, A. Shrier, and J. Belair, Chaos, edited by A. V. Holden (Manchester University Press, Manchester, 1986), pp. 237-256.

[2] R.G. Turcott and M.C. Teich, Ann. Biomed. Eng. 24, 269 (1996).

[3] C.D. Wagner and P.B. Persson, Cardiovasc. Res. 40, 257 (1998); Nature (London) 383, 323 (1996).

[4] P.Ch. Ivanov, M.G. Rosenblum, C.K. Peng, J. Mietus, S. Havlin, H.E. Stanley, and A. Goldberger, Nature (London) 383, 323 (1996).

[5] S. Thurner, M.C. Feurstein, and M.C. Teich. Phys. Rev. Lett.
80, 1544 (1998).

[6] L.S. Nunes Amaral, A.L. Goldberger, P.Ch. Ivanov, and H.E. Stanley, Phys. Rev. Lett. 81, 2388 (1998).

[7] A. Marrone, A.D. Polosa, G. Scioscia, S. Stramaglia, and A. Zenzola, Phys. Rev. E 60, 1088 (1999).

[8] J.P. Egan, Signal Detection Theory and ROC Analysis (Academic Press, New York, 1975).

[9] J.A. Swets, Science 240, 1285 (1988).

[10] S. Thurner, M.C. Feurstein, S.B. Lowen, and M.C. Teich, Phys. Rev. Lett. 81, 5688 (1998). 